Meta

Journal des traducteurs

Translators' Journal

\section{BALLARD, Michel (2013) : Histoire de la traduction. Repères historiques et culturels. Bruxelles : De Boeck Supérieur, 234 p.}

\section{Marie-Alice Belle}

Volume 61, numéro 2, août 2016

URI : https://id.erudit.org/iderudit/1037769ar

DOI : https://doi.org/10.7202/1037769ar

Aller au sommaire du numéro

\section{Éditeur(s)}

Les Presses de l’Université de Montréal

\section{ISSN}

0026-0452 (imprimé)

1492-1421 (numérique)

Découvrir la revue

Citer ce compte rendu

Belle, M.-A. (2016). Compte rendu de [BALLARD, Michel (2013) : Histoire de la traduction. Repères historiques et culturels. Bruxelles : De Boeck Supérieur, 234 p.] Meta, 61(2), 479-481. https://doi.org/10.7202/1037769ar
Tous droits réservés C Les Presses de l’Université de Montréal, 2016

Ce document est protégé par la loi sur le droit d'auteur. L'utilisation des services d'Érudit (y compris la reproduction) est assujettie à sa politique d'utilisation que vous pouvez consulter en ligne.

https://apropos.erudit.org/fr/usagers/politique-dutilisation/
Cet article est diffusé et préservé par Érudit.

Érudit est un consortium interuniversitaire sans but lucratif composé de l'Université de Montréal, l'Université Laval et l'Université du Québec à Montréal. Il a pour mission la promotion et la valorisation de la recherche. https://www.erudit.org/fr/ 


\section{DOCUMENTATION}

\section{Comptes rendus}

Ballard, Michel (2013): Histoire de la traduction. Repères historiques et culturels. Bruxelles: De Boeck Supérieur, 234 p.

En 1992 paraissait la première édition de De Cicéron à Benjamin. Traducteurs, traductions, réflexions, du traductologue et historien de la traduction Michel Ballard. Réédité en 1995 et 2007, cet ouvrage pionnier se distinguait des autres premières synthèses historiques parues à l'époque ${ }^{1}$ dans la mesure où il proposait une histoire conceptuelle de la traduction alliant la documentation historique à une réflexion approfondie sur le développement et la confrontation de différentes approches théoriques de la traduction en Europe, de l'Antiquité au début du XX $\mathrm{X}^{\mathrm{e}}$ siècle. Publié dans la collection «Traducto» des presses universitaires belges De Boek Supérieur, le volume Histoire de la Traduction. Repères historiques et culturels offre à la fois un supplément et un prolongement didactique à De Cicéron à Benjamin. La période couverte et la structure historique demeurent les mêmes: l'ouvrage est divisé en cinq chapitres, couvrant respectivement l'Antiquité, le MoyenÂge, la Renaissance, l'Âge classique et les Lumières, et un long XIX ${ }^{e}$ siècle ("Des Lumières à l'aube du XXe siècle»). Deux modifications majeures sont cependant à noter. La première concerne le format du volume, qui reprend la formule pédagogique propre aux ouvrages de la collection «Traducto» de De Boek Supérieur: division des chapitres en sections et sous-sections facilement repérables grâce à divers procédés de mise en page; insertion, en marge du texte, d'encadrés offrant des études de cas concises et des mises au point conceptuelles; présence systématique, en fin de chapitre, d'une section de synthèse intitulée "Faites le point», d'une bibliographie thématique propre à chaque période («Pour aller plus loin»), et d'un riche assortiment de questions et de pistes de réflexion sur les thèmes abordés dans chaque chapitre («Testez vos connaissances»). Le second apport de l'ouvrage consiste en une extension de l'aire géographique originellement couverte, en particulier aux chapitres 4 et 5 qui incluent des sections consacrées aux pays de langues slaves, à la (proto-)Roumanie et aux pays nordiques.

Les objectifs de l'ouvrage sont clairement exposés dans l'avant-propos (p. 7). S'inscrivant explicitement dans le mouvement récent de valorisation de l'histoire de la traduction comme sousdiscipline des études traductologiques ${ }^{2}$, Ballard rappelle l'importance de la composante historique dans la formation des traducteurs et dans la constitution de la traductologie comme un champ d'études à part entière. Ainsi, déclare-t-il, si la pensée sur la traduction semble s'être longtemps caractérisée par un certain «éparpillement» théorique ${ }^{3}$, les traducteurs ne parlant le plus souvent qu'en leur nom propre et sans nécessairement développer une "conscience historique» de leur pratique, il revient à l'historien de dégager les tendances, les composantes et les enjeux de la traduction comme pratique linguistique, mais aussi sociale, esthétique, philosophique et éthique. L'ouvrage propose donc d'établir les «repères» nécessaires à une vision d'ensemble, selon les principes de l'approche descriptive (curieusement nommée «traductologie réaliste (non prescriptive)» en quatrième de couverture), et en se limitant principalement au territoire européen. Les liens avec l'histoire théorique sont aussi clarifiés: si cette dernière ne représente pas l'objet principal du volume, elle peut se lire pour ainsi dire en filigrane, et participe au «balisage» historique. Filant abondamment la métaphore cartographique, Ballard souligne enfin le caractère à la fois initiatique et encyclopédique de l'ouvrage, dont l'objectif est donc de «donner les moyens à celui [et celle, faudrait-il ajouter] qui veut prendre contact avec ce champ, mal connu et immense, de le parcourir à grands pas avec une carte» (p. 7).

Le guide d'orientation se révèle extrêmement riche et détaillé. Les jalons historiques, on l'a dit, reprennent ceux, «classiques [et] assez largement admis» (p. 7), déjà adoptés dans De Cicéron à Benjamin. Les chapitres sont eux-mêmes organisés selon une logique assez variable: le second, consacré au Moyen-Âge, suit un ordre plutôt chronologique, tandis que celui sur la Renaissance dévoue des sections entières à l'imprimerie et à la traduction des textes sacrés. Dans la plupart des cas, la subdivision se fait par aires géographiques et linguistiques, selon une logique qui ne va pas sans rappeler celle des "domaines» de Valery Larbaud: ce dernier est d'ailleurs invoqué dès les premières lignes de l'avant-propos, et se voit consacrer une page entière (p. 189). Les variations dans l'organisation des chapitres sont parfois un peu problématiques. Par exemple, comme la question de la traduction biblique est renvoyée à la fin du chapitre 3 , on trouve à peine mention de Luther 
dans la section "pays de langue allemande», qui se trouve par le fait même réduite à quelques paragraphes. Par ailleurs, la traduction des Mille et Une Nuits de Galland est abordée dans la section du chapitre 4 spécifiquement consacrée à "l'Orient»; mais celles produites par la génération suivante de traducteurs anglais, allemands et français des Nuits sont traitées séparément, au sein des sections respectivement consacrées à l'Allemagne, à l'Angleterre et à la France. Si le recours à l'index est donc nécessaire pour qui cherche à retrouver une information spécifique, l'exploration des aires spatiolinguistiques dessinées dans l'ouvrage est tout de même facilitée par le système de titres et de sous-titres qui permet de distinguer les sous-domaines abordés, mais aussi de repérer les acteurs majeurs (Jérôme, Ibn Ishaq, d'Ablancourt), d'identifier les notions-clés (les «belles infidèles»), ainsi que les débats importants et les évolutions majeures dans les conceptions et les pratiques de la traduction sur le long terme.

Dans le pari presque intenable qui consiste à réunir de manière ordonnée une matière par nature foisonnante, les divers encadrés qui ponctuent le texte représentent un atout majeur. Ces derniers présentent comme en gros plan certaines figures importantes de la traduction, tels Ibn Ishaq, Florio, Goethe, etc. Mais ils permettent aussi d'attirer l'attention sur des phénomènes peut-être moins connus mais tout aussi significatifs, comme la pratique de la traduction indirecte en Angleterre à la Renaissance; l'importance de la médiation italienne pour les humanistes français; ou encore l'histoire des différentes versions d'Alice in Wonderland ou de Madame Bovary en Europe. Une autre réussite du volume est la réunion, à la fin de chaque chapitre, de pistes bibliographiques et de questions de réflexion aussi riches que pertinentes. Ces dernières, qui permettent non seulement de revenir sur les éléments abordés dans le chapitre, mais aussi d'amorcer une véritable réflexion théorique et critique, vont bien au-delà du «test de connaissances" annoncé en titre de section.

Il est par conséquent assez étonnant de voir le peu de place consacré à certains aspects idéologiques et politiques de la traduction. Peut-être expliquera-t-on ce choix par une interprétation restreinte de l'approche descriptive (ou « réaliste»), mais il semble presque naïf, après les travaux d'Edward Saïd et de tant d'autres, de présenter des sections sur «l'Orient» ou les «traductions orientales» sans rappeler les procédés de projection culturelle, parfois fantasmatiques, et souvent idéologiquement problématiques, qui procèdent à la construction de cet espace imaginaire. Certes, Ballard indique les aspects "multiples» de la réception des textes "orientaux", et indique comment «l'antagonisme violent, agressif, commence à se transformer en fascination»(p. 146); mais les exemples qu'il en propose renvoient presque exclusivement au cas des Mille et Une Nuits donnant ainsi raison, comme a posteriori, aux propos d'Anquetil-Duperron lorsqu'il soulignait, dès les années 1770, les limites et les dangers d'une représentation de l'«Orient» qui se limiterait aux Nuits de Galland ${ }^{4}$.

L'ouvrage passe d'ailleurs presque entièrement sous silence le rôle de la traduction et de l'interprétation dans l'histoire coloniale. Les Jésuites sont mentionnés à propos du programme de formation d'interprètes lancé par Colbert (p. 147-148), mais on ne trouve aucune trace des travaux de grammaire, de lexicographie et de traduction issus de la présence de missionnaires européens dans les colonies. On pensera par exemple, dans le contexte des Amériques, aux remarques sur la diversité des langues et les difficultés de la traduction dans les Relations des Jésuites de la Nouvelle France de Le Jeune (XVII ${ }^{\mathrm{e}}$ siècle); aux traductions des textes de la tradition Maya par le missionnaire espagnol Ximénez au XVIII ${ }^{\mathrm{e}}$ siècle, et par Brasseur de Bourbourg au siècle suivant. De même, la contribution des philologues anglais, allemands et français à l'établissement et à la traduction des textes sanskrits appartient sans doute à l'histoire coloniale de l'Inde; mais on ne saurait oublier que ces traducteurs et leurs lecteurs étaient tous en grande majorité européens. Leurs travaux, diffusés par l'entremise des sociétés savantes et des journaux spécialisés, ont ainsi contribué, au même titre que ceux des Romantiques (qui faisaient d'ailleurs aussi partie de leur lectorat), à la réflexion européenne sur la traduction comme confrontation avec l'Autre culturel.

Ballard justifie la place centrale ainsi donnée à l'Europe, à l'exclusion totale de l'Amérique, de l'Asie et de l'Afrique, en avançant que, jusqu'au $\mathrm{XX}^{\mathrm{e}}$ siècle, l'Europe reste dans un "processus, normal, d'échange et de brassage à son propre niveau» (p. 210). On trouvera peut-être une autre explication au choix d'une perspective strictement eurocentrique dans le contexte de la formation actuelle des traducteurs en Europe, qui vise en bonne partie à répondre aux besoins des institutions européennes. Ainsi, l'espace consacré dans le volume aux pays d'Europe de l'Est reflète sans aucun doute leur entrée récente dans l'Union. Cette orientation n'enlève d'ailleurs rien à la richesse des informations proposées, et permet de mettre en lumière des pans par ailleurs méconnus de la circulation européenne des textes.

Par la précision des informations, présentées dans une langue à la fois nuancée et accessible, et par l'effort d'organisation et de structuration des données, l'ouvrage offre de fait aux étudiants de premier cycle un "guide» plus facile à manier que 
l'Histoire de la traduction en Occident de Van Hoof ou que Les traducteurs dans l'histoire de Delisle et Woodsworth. Les enseignants et étudiants des cycles supérieurs tireront plus particulièrement profit des éléments de bibliographie thématique et des pistes de réflexion proposées en fin de chapitre. Les limites thématiques de l'ouvrage et sa structure, qui se prête mal au calendrier universitaire nord-américain, rendent malaisé son usage comme manuel d'enseignement dans le contexte canadien. Mais il représente sans aucun doute un ouvrage de référence précieux pour qui cherche à découvrir, approfondir ou faire connaître les éléments majeurs de la tradition européenne dans l'histoire de la traduction.

Marie-Alice Belle

Université de Montréal, Montréal, Canada

\section{NOTES}

1. On pensera en particulier, pour le domaine francophone, à l'Histoire de la traduction en Occident d'Henri Van Hoof (1991) ou au collectif dirigé par Jean Delisle et Judith Woodsworth, Les traducteurs dans l'histoire, dont la première édition date de 1995 . Voir aussi Santoyo 1999.

2. Voir sur ce point l'introduction au collectif Charting the Future of Translation History (Bastin et Bandia 2006: 1-3), et plus récemment les propos de L. D'hulst dans l'introduction de ses Essais d'histoire de la traduction (2014: 7-14).

3. Ballard renvoie ici à Mounin, mais la question de l'éparpillement théorique et critique s'est posée plus récemment à propos de la méthode traductologique en tant que telle. Voir par exemple Pym 1992.

4. Voir en particulier les remarques d'A.-H. Anquetil Duperron dans sa Législation Orientale (1778: 293), citées dans Berthier 2005: 361.

\section{RÉFÉRENCES}

Bastin, Georges L. et Bandia, Paul, dirs. (2006): Charting the Future of Translation History. Ottawa: Les Presses de l'Université d'Ottawa.

Berthier, Annie (2005): Un lapin dans la lune: autour de la réception et de la traduction des œuvres orientales en France. D'un Orient l'autre: Actes des troisièmes journées de l'Orient (Société Asiatique). Paris-Louvain: Peeters, 351-363.

Delisle, Jean et Woodsworth, Judith, dirs. (1995): Les Traducteurs dans l'Histoire. Ottawa: Presses de l'Université d'Ottawa.

D'Hulst, Lieven (2014): Essais d'histoire de la traduction. Avatars de Janus. Paris: Classiques Garnier.

PyM, Anthony (1992): Complaint Concerning the Lack of History in Translation History, Livius 1:1-11.
SAntoyo, Julio Cesar (1999): Historia de la traducción: quince apuntes. León: Universidad de León.

Van Hoof, Henri (1991): Histoire de la Traduction en Occident: France, Grande-Bretagne, Allemagne, Russie, Pays-Bas. Paris: Duculot.

House, Juliane (2015): Translation quality assessment: past and present. Londres: Routledge, $160 \mathrm{p}$.

Au fil de la deuxième moitié du XXe siècle, l'évaluation de la qualité des traductions est devenue une spécialisation à part entière au sein de la traductologie (Lee-Jahnke 2001: 206). Juliane House, professeure émérite de l'Université de Hambourg, en a d'ailleurs fait son principal champ de recherche, elle qui est l'auteure d'un des modèles d'évaluation les plus reconnus de la discipline. Son dernier ouvrage fournit un panorama des plus récentes recherches interdisciplinaires portant sur la traduction, les études de corpus, les études psycho- et neurolinguistiques, ainsi que sur la communication interculturelle et la mondialisation. Ceci lui sert d'assise pour réviser son modèle fonctionnel-pragmatique au goût du jour. Comme ses prédécesseurs, décrits respectivement dans A model for translation quality assessment (1977/1981) et Translation quality assessment: a model revisited (1997), cette nouvelle édition s'appuie sur une analyse et une comparaison détaillées des caractéristiques textuelles et culturelles des textes source et cible. Or, pour House, l'évaluation de la qualité est plus qu'une application pratique. $\mathrm{Au}$ contraire, elle est intrinsèquement liée à une théorie de la traduction:

Evaluating the quality of a translation presupposes a theory of translation. Thus different views of translation lead to different concepts of translational quality, and hence different ways of assessing quality (House 1997: 1).

Le premier chapitre de Translation quality assessment: past and present est d'ailleurs consacré à des questions théoriques. House s'inquiète de voir la traductologie envisager de plus en plus la traduction comme un phénomène principalement social, culturel, politique, éthique et idéologique. Elle affirme que, malgré l'importance de ces divers aspects, il ne faut pas perdre de vue que la traduction demeure essentiellement un acte linguistique. En effet, elle définit la traduction comme une opération linguistique-textuelle par laquelle un texte dans une langue donnée est recontextualisé dans une autre langue, c'est-à-dire le remplacement d'un texte par un autre. L'auteure reconnaît cependant que la langue est imprégnée d'éléments culturels 